\title{
Methadone for Opioid Use Treatment during Pregnancy: Trends in Postpartum Dose Adjustments
}

\author{
Clara Ward, MD ${ }^{1}$ Carl W. Christensen, MD, $\mathrm{PhD}^{2}$ \\ ${ }^{1}$ Division of Maternal-Fetal Medicine, Department of Obstetrics, \\ Gynecology, and Reproductive Sciences, University of Texas Health \\ Science Center/McGovern Medical School, Houston, Texas \\ 2 Department of Obstetrics and Gynecology, Wayne State University, \\ Detroit, Michigan
}

\begin{abstract}
Address for correspondence Clara Ward, MD, Division of Maternal Fetal Medicine, Department of Obstetrics, Gynecology, and Reproductive Sciences, University of Texas Health Science Center/ McGovern Medical School, 6431 Fannin, MSB 3.286, Houston, TX 77005 (e-mail: clara.ward@uth.tmc.edu).
\end{abstract}

Am J Perinatol Rep 2020;10:e202-e209.

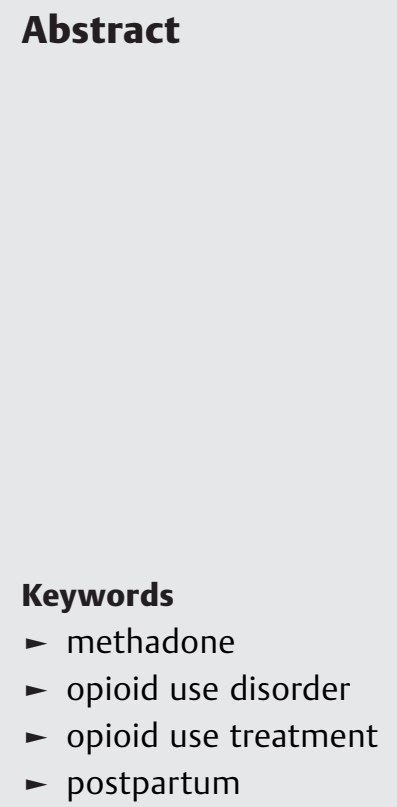

Objective This study examines methadone dose adjustment postpartum. Methods A retrospective study of women with methadone for opioid use treatment (OUT) during pregnancy was performed. Patient charts were reviewed and data were extracted. Methadone doses from five temporal data points for each patient were used: starting dose, day of delivery, and 1, 2, and 6 months postpartum.

Results Over 26 months, 49 pregnancies to women using methadone for OUT were evaluated and 20 (41\%) were included. The mean methadone starting dose was $47 \mathrm{mg}$, compared with $86 \mathrm{mg}$ at the time of delivery. The mean dose postpartum remained unchanged from delivery and $75 \%$ of pregnancies required the same dose or higher 1 month postpartum. By 2 months postpartum, only 33\% were able to decrease their methadone dose. Twelve pregnancies completed follow-up until 6 months postpartum; only $17 \%$ of patients were able to decrease their dose, with an overall mean dose decrease was $12 \%$. There was no difference between the mean dose at delivery and the 6-month postpartum dose.

Conclusion Patients using methadone for OUT during pregnancy achieved minimal dose decreases postpartum. Patients should be counseled that postpartum dose tapers may be challenging and about alternatives to methadone for OUT.
The management of opioid use disorder (OUD) in pregnancy remains a significant perinatal problem, particularly as the nonmedical usage of prescription opiates augments heroin abuse. Despite the well-established practice of methadone for OUD for patients during pregnancy, evidence-based guidelines defining appropriate dosing remain elusive. Information on methadone dosing patterns during pregnancy has centered on neonatal outcome, specifically, whether maternal dose impacts the prevalence of neonatal abstinence syndrome. $^{1-6}$

Pregnancy-induced physiologic changes are thought to affect methadone metabolism and body distribution, increasing third trimester methadone requirements. ${ }^{7,8}$ As women's bodies regain their prepregnancy characteristics after delivery, one would expect that their medications may

received

March 30, 2020

accepted after revision

April 6, 2020 be lowered to reflect these presumably reduced needs. In fact, earlier guidelines suggest women treated during pregnancy may reduce their dose to their prepregnancy regimen or half of their third trimester dose. However, this recommendation was supported primarily by clinical opinion and experience due to a paucity of evidence-based research. ${ }^{9,10}$

The perception is that once the metabolic changes of pregnancy resolve postpartum, patients can resume their prior opioid use treatment trajectory, whether that is a return to her pre-pregnancy methadone dose or other techniques. Current observations reveal that this clinical tradition may not be appropriate nor feasible. In the first identified study to determine dose adjustments pre- and postdelivery in patients enrolled in a clinical trial to evaluate buprenorphine and methadone for OUT during pregnancy,
Copyright @ 2020 by Thieme Medical Publishers, Inc., 333 Seventh Avenue, New York, NY 10001, USA. Tel: $+1(212) 760-0888$.
License terms

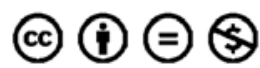


medication doses were stable during the peripartum, and there were no dose adjustments in the postpartum period. ${ }^{11}$ Only recently have studies begun to build on this finding to assess the maternal impact of dose changes. ${ }^{12-14}$

The objective of this study was to evaluate postpartum dose adjustments in women undergoing methadone for OUT during pregnancy.

\section{Methods}

The Jefferson Avenue Research Clinic (JARC) in Detroit, Michigan, associated with the Wayne State University Department of Psychiatry, is home to several research programs committed to improving care for patients with substance abuse. The Department of Obstetrics and Gynecology has a specialized prenatal care clinic, the Eleonore Hutzel/James Wardell Recovery Center, dedicated to the care of women with substance use disorder, as well as chronic pain, in pregnancy. These two entities have united in the antenatal care of women with OUD treated at the study institution.

Our retrospective observational case series was approved by the Wayne State University Institutional Review Board. All patients with a singleton pregnancy using methadone for OUT at JARC between December 2006 and February 2009 were evaluated and all pregnant patients during this period were identified. Patients were subsequently excluded secondary to enrollment in the MOTHER (Maternal Opioid Therapy: Human Experimental Research) study (discussed later), detoxification and discharge from the clinic because of nonadherence, known buprenorphine replacement therapy, multiple gestation, or insufficient data. Patients who delivered at another institution were excluded, unless gestational age at delivery and demographic information were available. Patients initiating methadone for OUT postpartum, transferred from other methadone clinics where dosing history was not available, or those lost to follow up postpartum were also excluded.

The MOTHER study is a randomized double-blind control trial comparing methadone and buprenorphine conducted by the National Institutes of Health. The reason for excluding these patients is two folds. First, the study arms at our institution were blinded, thus whether the patients were taking methadone or buprenorphine was unknown. Second, only early antenatal and postpartum methadone doses are available, due to enrollment in the study, precluding analysis according to the terms of our study. Due to the limited data available and to homogenize our study population, these patients were excluded.

The protocol for nonadherence after delivery is the same as that for nonpregnant patients at JARC: patients who continue to use illicit substances despite counseling, increased levels of care, and abstinence contracts will receive tapers in their methadone dose of $5 \mathrm{mg}$ in every 2 days until they reach a dose of $0 \mathrm{mg}$. These patients are referred to residential treatment before the taper has begun. This is not representative of the usual postpartum dose adjustments, hence these patients were excluded.
The primary outcome of this study was change in methadone dose adjustment postpartum. To ascertain this, we selected five-methadone dose data points for each patient: starting dose, dose on the day of delivery, and doses at 1,2 , and 6 months postpartum were obtained. Starting dose refers to the dose at the time of enrolment. Two patients were lost to follow-up prior to their 2- month postpartum visit, and a total of eight patients were lost to follow-up prior to their 6-month postpartum visit.

Criteria for a dose increase were subjective complaints of withdrawal symptoms. Antepartum methadone doses were increased in increments of $5 \mathrm{mg}$ until relief of symptoms was achieved. For pregnant patients, physical documentation of withdrawal symptoms was not required. Patients requesting multiple increases were observed for 3 hours after dose administration to exclude excessive sedation, as judged by the physician.

Postpartum dose tapers were similarly attempted in 5-mg increments as tolerated by the patient. Smaller dose intervals were used when deemed clinically necessary. Dose adjustments were made by only two physicians with similar clinical practices in the management of pregnant women receiving methadone for OUT.

Statistical analysis was performed using SPSS version 15.0 (Chicago, IL). To examine the change in methadone maintenance dose, the dose on day of delivery is expressed as a percentage of the starting dose for each patient. The 1-, 2-, and 6-month postpartum doses are expressed as percentages of both the starting dose and the delivery dose for examining postpartum changes. Categorical variables including race, mode of delivery, hepatitis $\mathrm{C}$ status, concomitant substance abuse, and addiction to heroin or prescription opiates were analyzed using Pearson's Chi-square and Fisher's exact tests. Continuous variables, including age, parity, gestational age at delivery, birth weight, and placental weight were analyzed using Student's independent samples $t$-test. Statistical significance is determined as $p<0.05$ and all statistical tests are two-tailed.

\section{Results}

There were 49 pregnancies of women using methadone for OUT due to OUD during the 2-year study period. Out of these, 29 patients were excluded as follows: 10 (34\%) were lost to follow-up (three delivered at our institution, seven at other institutions), 7 patients (24\%) had insufficient data for analysis, 9 (31\%) were participants in the MOTHER study, and 1 patient (3\%) underwent a methadone taper postpartum as a discharge protocol from the clinic due to nonadherence. One patient had a twin gestation (3\%), and one was maintained on buprenorphine (3\%; - Fig. $\mathbf{1}$ ).

A total of 20 pregnancies, belonging to 19 women, remained for analysis. Out of these, 16 deliveries occurred at our institution and complete demographic and clinical data were available. The study group was predominantly Caucasian (85\%) with a mean age of 28 years (range: $21-37$ y). Seventy-five percent of the patients were multiparous with a mean of 2.5 deliveries prior to the index pregnancy (range: 1-4). Forty-two percent of patients were positive for 


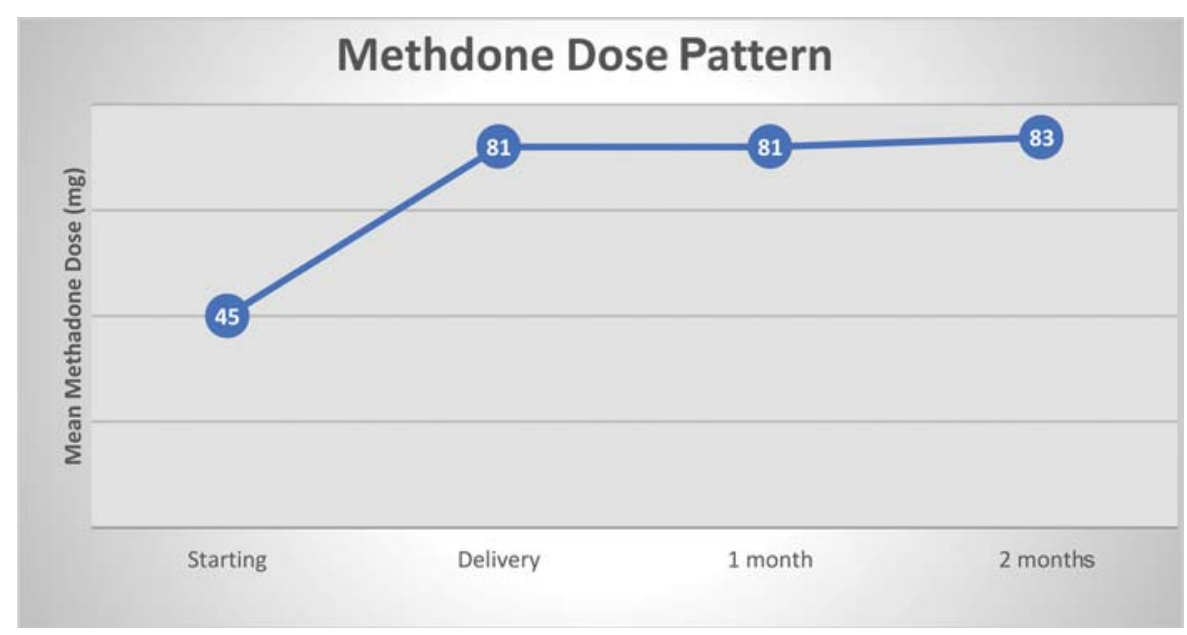

Fig. 1 Methadone dose pattern.

hepatitis C and 75\% were tobacco dependent. For all 19 patients, the primary substance of addiction was identified: heroin $(58 \%)$, prescription opioids $(32 \%)$, or both $(10 \%)$. The mean duration of opioid use disorder was 8 years (range: 1-19 years; - Table $\mathbf{1}$ ).

Delivery data were available on $80 \%(n=17)$ of pregnancies. The majority of pregnancies $(88 \%)$ delivered at term with a mean gestational age of $38^{5 / 7}$ weeks (range: $34^{6 / 7}-41$ 2/7 weeks). None of the patients had iatrogenic preterm deliveries. The cesarean section rate was $31 \%$; $40 \%$ were performed secondary to nonreassuring fetal status. Neonatal data were available on $81 \%$ of infants; $85 \%$ had Apgar's scores $\geq 8$ at 1 and 5 minutes. Only one neonate was small for gestational age, defined as $<10$ th percentile for gestational age. Only one infant had abnormal umbilical cord gases ( $\mathrm{pH}=6.88$; base excess, -21 ); this infant also was one of two with an Apgar's score below 8 at 1 minute. This pregnancy was delivered by emergency cesarean section secondary to fetal bradycardia, active cocaine use and placental

Table 1 Maternal demographics and characteristics

\begin{tabular}{|l|l|}
\hline & $n /$ total (\%) or (range) \\
\hline Age (y) & $28(21-37)$ \\
\hline Parity & $2.5(2-4)$ \\
\hline Nulliparous & $4 / 16(25)$ \\
\hline Race & \\
\hline Caucasian & $16 / 19(85)$ \\
\hline African American & $3 / 19(15)$ \\
\hline Hepatitis C positive & $8 / 19(42)$ \\
\hline Tobacco dependence & $12 / 16(75)$ \\
\hline Primary substance of addiction & \\
\hline Heroin & $11 / 19(58)$ \\
\hline Prescription opioids & $6 / 19(31)$ \\
\hline Both & $2 / 19(11)$ \\
\hline Duration of addiction & $8(1-19)$ \\
\hline
\end{tabular}

abruption. The mean infant birthweight was 2,874 $\mathrm{g}$ (range: 2,180-3,775 g; - Table 2).

Antepartum, day of delivery, and at least one postpartum methadone dose were available on all pregnancies included in the study. Data were complete on all pregnancies with the exception of two, which were lost to follow-up prior to the 2-month postpartum endpoint. All other data collection points on these two pregnancies were included in the analysis. Twelve pregnancies had complete follow-up until 6 months postpartum.

The mean dose at delivery was $86 \mathrm{mg}$ (range: $50-195 \mathrm{mg}$ ). This represents an increase of $83 \%$ from the starting methadone dose (mean of $47 \mathrm{mg}$ [range: $30-85 \mathrm{mg}$ ]; $p=0.001$ ). Mean methadone doses remained stable at 1,2, and 6 months postpartum (mean; 85,88 , and $89 \mathrm{mg}$, respectively, [range: 36-195 mg]). Similarly, there was no change between delivery dose and postpartum doses $(100,104$, and $107 \%$ for 1,2 , and 6 months, respectively; $p=0.269$; - Table 3 ).

Of them, $60 \%$ pregnancies required the same methadone dose 1-month postpartum as on the day of delivery; moreover, three additional patients (15\%) required medication increases. In these three patients, the mean dose increase was $11 \mathrm{mg}$, representing an increase of $17 \%$ (range: $3-30 \%$ ). In only four pregnancies (20\%), a dose decrease was achieved at 1 -month postpartum. The mean dose decrease was $9 \mathrm{mg}$ (range: 3-20 mg), which represented a decrease of 9\% (range: $5-14 \%)$.

By 2-months postpartum, a total of six pregnancies (6/18; $33 \%)$ had decreased their dose. Three of these patients further decreased their dose from 1-month postpartum, one experienced no further decrease from 1-month postpartum, and for another two patients this was their first postpartum decrease (one had previously increased at the 1-month data point). Four patients required new increments in their methadone doses at 2-month postpartum (22\%). Overall, six patients (30\%) had a net increase in their methadone dose postpartum, six (30\%) were able to achieve a decrease, and in eight patients (40\%), the dose remained the same. In the pregnancies in which a postpartum decrease ensued, the mean dose decrease was $10 \mathrm{mg}$ (range: 3-20 mg) 
Table 2 Clinical characteristics

\begin{tabular}{|l|l|}
\hline & n/total (\%) \\
\hline Gestational age at delivery & \\
\hline Term & $14 / 16(88)$ \\
\hline Preterm & $2 / 16(12)$ \\
\hline Mean (wk) & $38^{5 / 7}$ \\
\hline Range (wk) & $34^{6 / 7}-41^{2 / 7}$ \\
\hline Mode of delivery & \\
\hline SVD & $11 / 16(69)$ \\
\hline Cesarean section & $5 / 16(31)$ \\
\hline Apgar's scores & \\
\hline 8 or 9 & $11 / 13(85)$ \\
\hline$<8$ & $2 / 13(15)$ \\
\hline Birth weight & \\
\hline AGA & $12 / 13(92)$ \\
\hline SGA & $1 / 13(8)$ \\
\hline Mean (g) & 2,874 \\
\hline Range (g) & $2,180-3,775$ \\
\hline Umbilical cord gas & $1 / 13(8)$ \\
\hline$\geq 7.1$ & \\
\hline$<7.1$ & \\
\hline
\end{tabular}

Abbreviations: AGA, appropriate for gestational age; SGA, small for gestational age; SVD, spontaneous vaginal delivery.

Table 3 Dosing patterns

\begin{tabular}{|l|l|l|l|l|}
\hline Time & $\begin{array}{l}\text { Mean } \\
\text { dose } \\
(\mathrm{mg})\end{array}$ & $\begin{array}{l}\text { Range } \\
(\mathrm{mg})\end{array}$ & $\begin{array}{l}\text { Percentage } \\
\text { change }\end{array}$ & $p$-Value \\
\hline Starting dose & 45 & $30-70$ & - & - \\
\hline Delivery & 81 & $35-195$ & $189^{\mathrm{a}}$ & 0.001 \\
\hline 1-month PP & 81 & $35-195$ & $100^{\mathrm{b}}$ & $0.5^{\mathrm{c}}$ \\
\hline 2-months PP & 83 & $25-192$ & $104^{\mathrm{b}}$ & $0.45^{\mathrm{d}}$ \\
\hline
\end{tabular}

Abbreviation: PP, postpartum.

a Starting dose set at $100 \%$.

b Date of delivery dose set at $100 \%$.

${ }^{\mathrm{C}} 1$-month postpartum dose compared with date of delivery dose.

${ }^{\mathrm{d}} 2$-month postpartum dose compared with date of delivery dose.

that represents a mean percentage decrease from the dose at delivery of $10 \%$ (range: $2-14 \%$; - Table 2 ).

Of the 12 remaining patients at 6 -month postpartum, 4 (33\%) experienced a decrease in their methadone dose from delivery. All of these patients also had a dose decrease at 2month postpartum. The mean dose decrease was $13 \mathrm{mg}$ (range: $8-18 \mathrm{mg}$ ), representing a mean percentage decrease from the dose at delivery of $13 \%$ (range: $8-21 \%$ ). Seven patients required increases from their delivery dose, while one remained at the same dose. Overall, for all pregnancies included in the study, six successfully tapered their dose (mean, $14 \mathrm{mg}$ [range: $5-27 \mathrm{mg}$ ]; mean, $15 \%$, [range: $8-31 \%$ ]), five tolerated no changes from their delivery dose (range: 60--
Table 4 Comparison of patients with dose decrease versus no decrease: demographics

\begin{tabular}{|c|c|c|c|}
\hline & $\begin{array}{l}\text { Dose decrease } \\
n=6(\%)\end{array}$ & $\begin{array}{l}\text { No decrease } \\
n=13(\%)\end{array}$ & $p$-Value \\
\hline \multicolumn{3}{|l|}{ Race } & \multirow[t]{3}{*}{0.53} \\
\hline Caucasian & $6(100)$ & $10(77)$ & \\
\hline African American & $0(100)$ & $3(23)$ & \\
\hline \multicolumn{3}{|l|}{ Age } & \multirow[t]{2}{*}{0.04} \\
\hline Mean $(y)$ & 25 & 30 & \\
\hline \multicolumn{3}{|l|}{ Parity } & \multirow[t]{4}{*}{0.76} \\
\hline Nulliparous & $3(50)$ & $1(8)$ & \\
\hline Multiparous & $3(50)$ & $12(92)$ & \\
\hline Mean & 0.8 deliveries & 1.7 deliveries & \\
\hline \multicolumn{3}{|l|}{ Hepatitis C } & \multirow[t]{3}{*}{0.64} \\
\hline Positive & $3(50)$ & $5(38)$ & \\
\hline Negative & $3(50)$ & $8(62)$ & \\
\hline \multicolumn{3}{|l|}{$\begin{array}{l}\text { Tobacco } \\
\text { dependence }\end{array}$} & \multirow[t]{3}{*}{0.73} \\
\hline Dependent & $5(83)$ & $10(77)$ & \\
\hline Not dependent & $1(17)$ & $3(23)$ & \\
\hline \multicolumn{4}{|l|}{ Primary substance } \\
\hline Heroin & $5(83)$ & $6(46)$ & \multirow[t]{3}{*}{0.52} \\
\hline Prescription & $0(0)$ & $6(46)$ & \\
\hline Both & $1(17)$ & $1(8)$ & \\
\hline \multicolumn{3}{|l|}{$\begin{array}{l}\text { Duration } \\
\text { of addiction }\end{array}$} & \multirow[t]{2}{*}{0.22} \\
\hline Mean $(y)$ & 5.8 & 7.7 & \\
\hline \multicolumn{3}{|l|}{ BMI } & \multirow[t]{2}{*}{0.38} \\
\hline Mean & 30.5 & 33.5 & \\
\hline
\end{tabular}

Abbreviation: BMI, body mass index.

$120 \mathrm{mg}$ ), and nine experienced net methadone dose increases (mean, $13 \mathrm{mg}$ [range: $1-30 \mathrm{mg}$ ]; mean, 19\% [range: 3-38\%]).

The one patient in whom two pregnancies were completed during the study period (delivery dates were $\sim 13$-month apart) steadily increased her dose over the course of the first pregnancy and had an increase at 1-month postpartum which remained stable until 6-month postpartum. At the time of enrollment during her second pregnancy, her methadone requirements were unchanged from 6-month postpartum. Her dose was then further increased over the course of the second pregnancy, remaining stable from delivery until 3-month postpartum (at which time she was lost to follow-up).

Patients who were able to achieve a dose decrease postpartum were then analyzed and compared with those who required the same or higher methadone doses as on the day of delivery. There were no significant demographic or clinical differences between the two groups ( $p>0.05$ for all; - Table 4 ).

\section{Discussion}

Accurate estimates of the burden of opiate use in pregnancy remain elusive. The National Survey on Drug Use and Health estimates that $4 \%$ of women in 2010 used illicit substances 
during pregnancy. ${ }^{10}$ However, even in 1998 prior to the recognition of the opioid epidemic, illicit drug use in pregnant women at this study institution approached $40 \%$ of pregnancies and nearly one-third of abuse was attributed to opioids. ${ }^{15}$ Only $11 \%$ of women in the study by Ostrea et al admitted to illicit drug use, highlighting the disparity between self-reported and actual use. As access to prescription opioids proliferated over the past two decades, a public health crisis has been acknowledged. While the number of women admitted to substance use treatment facilities over the past 20 years has remained stable at $4 \%$, the proportion of women among them who reported prescription opioid abuse increased from 2 to $28 \%$, with $19 \%$ identifying it as their primary substance of abuse. ${ }^{16}$ Indeed, from 1998 to 2008 , Pan and Yi found that births affected by opioids increased by one-third from 14 to $22 \%$ nationwide. ${ }^{17}$

The improvements in obstetric, neonatal, and social outcomes for women enrolled in methadone for OUT programs compared with those actively using illicit substances is well established. ${ }^{18-20}$ Alternatives to methadone for OUT exist, including antepartum inpatient detoxification, ${ }^{21,22}$ heroin and morphine assisted treatment, ${ }^{23,24}$ buprenorphine, ${ }^{25,26}$ and implantable delivery systems. ${ }^{27,28}$ Despite methadone for OUT being historically the most widely accepted therapeutic intervention, the scope of research has been relatively narrow. Concern for neonatal outcomes and the reduction of neonatal abstinence syndrome (NAS) has dominated the literature to date. Investigations of maternal dosing patterns have focused on whether or not there is a threshold dose at which NAS is either precipitated or avoided. ${ }^{3-6,29}$ While this is an extremely important consideration in caring for pregnant women with opioid use disorder, there remains a void in evidence-based understanding of current maternal management techniques.

Women have been observed to have lower plasma methadone concentrations than men, as well as higher urine metabolite to methadone ratios, suggesting that women have increased metabolism of methadone at baseline. Knowledge of fundamental physiologic alterations that occur with pregnancy that may affect the metabolism of methadone include hormonal induction of the cytochrome p450 system, increased volume of distribution, increased renal clearance, and the impact of the fetoplacental unit. ${ }^{8,30}$ Methadone is metabolized by the liver in both the nonpregnant and the gravid woman. However, during pregnancy, the placenta behaves as an extrahepatic source of enzymes that contribute to the conversion of methadone to its inactive metabolite. ${ }^{30}$ While the placenta and the fetal compartment are thought to comprise a minimal portion of gestational metabolism, ${ }^{30}$ it is still unclear the extent and the mechanism by which these new players exert their influence and contribute to the changes in metabolism seen during pregnancy. Another study sought to understand intragestational and interplacental variation in enzyme levels. ${ }^{31}$ They found that enzymatic activity increased as pregnancy progressed; however, the extent to which this occurred varied significantly from one placenta to another. Studies by Ahmed and colleagues $^{32}$ have suggested opioid tolerance at the placental level, with downregulation and desensitization of receptors in trophoblast tissue exposed to methadone in vitro. ${ }^{32,33}$

While these findings support evidence of increasing methadone requirements throughout pregnancy, ${ }^{7}$ they do not explain the persistence of elevated doses postpartum. Even if some of these changes persist for longer than the conventional postpartum period, one would expect that with the gradual restitution of a woman's normal physiology, there would be a progressive decrease in methadone requirements. Other published studies that chronicle the dosing trends postpartum have primarily had similar outcomes. The first such study, a secondary analysis of the MOTHER randomized control trial performed by Jones and colleagues reported postpartum dose adjustments. ${ }^{25}$ Their study included patients maintained on methadone or buprenorphine during pregnancy. Over the course of the 7-week study period, which included 3 weeks of antepartum and 4 weeks of postpartum, methadone or buprenorphine doses remained stable. This supports our study in which there was no net change between the methadone dose at delivery and doses at 1,2, and 6 months postpartum.

Recent studies have also investigated methadone dose changes. Albright et $\mathrm{al}^{12}$ focused on characterizing changes throughout gestation. However, they did note that $86 \%$ of patients were within $10 \mathrm{mg}$ of their delivery dose by 6 -week postpartum, with a mean decrease of $4 \mathrm{mg}$. Of note, they report that $24 \%$ of patients were able to return to their prepregnancy or starting doses, which contrasts with other studies. ${ }^{12}$ Bogen et $\mathrm{al}^{13}$ examined the pharmacokinetics of methadone during pregnancy and postpartum in 22 women. They reported that nearly one-third were able to decrease their methadone dose postpartum, with a mean decrease of $32 \mathrm{mg}$, which is significantly greater than in our and other studies. ${ }^{13}$ A larger study of 101 women found insignificant dose changes up to 3-month postpartum in $50 \%$ of women; the other $50 \%$ of women had either the same or higher doses postpartum. ${ }^{14}$

The most provocative finding of our study is the lack of a postpartum decrease in methadone requirements up to 6-month postpartum. Recommendations from the Center for Substance Abuse Treatment, Medication-Assisted Treatment for Opioid Addiction in Opioid Treatment Programs, Treatment Improvement Protocol Series no. 43 at the time of this study state that postpartum a woman may either resume prepregnancy doses or decrease her delivery methadone dose by $50 \%{ }^{34}$ These are based on the suggestions of current clinical practice unsubstantiated by empiric data and have not been borne out by subsequent investigations. ${ }^{28}$ Updated guidelines refrain from proposing a specific amount for postpartum titration, stress patient individuality, and acknowledge the inconclusive findings in existing literature. ${ }^{35}$

Our study supports the need for further evidence-based examination of postpartum methadone requirements. Seventy percent of the pregnancies in our study required the same dose or higher postpartum as on the day of delivery. For those that did achieve a dose decrease postpartum, the mean decrease was only $13 \%$, substantially less than the $50 \%$ decrease recommended by the Treatment Improvement Protocol Series, used at the time of this study. 
The prevailing physiologic and metabolic theories for pregnancy-induced dose changes fail to fully explain the difficulty of postpartum detoxification. The one patient with two pregnancies included in our study had no interval decrease in her methadone dose and continued to increase over the course of her second pregnancy. The possibility that pregnancy causes permanent or progressive alterations in the mechanism for methadone biotransformation is currently unexplored.

There is also the possibility of psychosocial or neurobiochemical changes that may impact the body's receptivity to the pharmacologic benefits of methadone. This study did not specifically assess the psychosocial stressors or environmental factors (outside of concomitant drug abuse) that may complicate new motherhood. Some studies have suggested that concomitant psychiatric disease, as well as concurrent tobacco dependence, makes methadone for OUT less successful. ${ }^{36}$ Additionally, the enhanced craving in pregnant women at baseline may go into "overdrive" in women with a history of addiction, in which the pathway for craving and pleasure is inherently altered. Whether or not these factors pose a challenge to methadone for OUT deserves further evaluation.

\section{Limitations and Strengths}

The three most apparent limitations of our study are its small sample size, homogenous population, and short duration of follow-up. While we have a large population of women with opioid use disorder in the metropolitan Detroit area, they fall primarily into three categories: those actively using illicit drugs and not enrolled in therapy; those enrolled in methadone clinics not affiliated with the university, and thus dosing schedules not as fastidiously recorded or accessible to researchers; and those at JARC who were included in this study. Due to the referral base of JARC, the patients are primarily Caucasian, while the patients in the first two groups may be more representative of the university teaching hospital patient population, which is approximately $80 \%$ African Americans.

Several patients were excluded to control for as many confounding factors as possible and to ensure completeness of available data. This limited our population available for study to 20 pregnancies. It is possible that no differences were observed in demographic and clinical parameters due to a lack of power. Our initial postpartum evaluation of dosing trends was limited to 2 months. The puerperal period is conventionally terminated at 6 weeks following delivery. While, we have overcome this landmark, it is possible that the physiologic derangements of pregnancy do not uniformly conform to this timeline. Consequently, we pursued dosage follow-up for 6 months in available patients. However, due to insurance considerations and rectification, our patient population was severely limited for long-term follow-up. Six months was chosen as a landmark to account for the thorough and adequate recession of the physiologic changes of pregnancy. Longer postpartum follow-up would enable us to determine if the inability to undergo detoxification postpartum is permanent or merely prolonged, and to what extent.
Despite these limitations, there are some unique aspects of our study in comparison to the published literature that deserve mention. First, all dose adjustments were performed by two licensed addiction physicians upon personal examination of the patient. This ensured clinical expertise, as well as consistency. Second, patients with concomitant use of benzodiazepines were excluded. It is well known that the simultaneous use of opioids and benzodiazepines predisposes patients to oversedation. It is unclear in the other studies that how this may have played a role in any dose decrements that were achieved. Third, we were able to examine the clinical obstetric features of these deliveries, as well as their association, or lack thereof with the ability to decrease the methadone dose postpartum. While the data are small, stressors, such as prematurity, surgical delivery, and parity, and their hormonal and psychosocial implications, may be areas of future study. While we were unable to assess the impact of obesity or postpartum weight retention and fat distribution in our study, that may also play a role in the inability to reduce the dose postpartum.

Our findings have the potential to affect clinical practice and research in several ways. First, it highlights the need for further investigation into the interaction between pregnancy and methadone, both at the molecular and human levels, to better understand the mechanism behind these findings. Second, we must change how we counsel our patients. Often, we reassure patients that their methadone doses will be titrated downwards postpartum, and that they will be able to undergo detoxification without difficulty. Our results show dismal success to this end. Third, if postpartum methadone requirements persist at higher levels longer than previously thought, we must ensure that broad spectrum resources for opioid use disorder treatment are available to patients for the duration required.

Recent recognition of the scope of opioid addiction has fueled renewed interest in treatment modalities during pregnancy. These outcomes, if confirmed, should spur the search for alternatives to methadone for the treatment of opioid use disorder in pregnancy. Studies suggest that buprenorphine may be equal or superior to methadone, ${ }^{25,26,37,38}$ in particular, regarding the severity of neonatal abstinence syndrome. ${ }^{39}$ Implantable naltrexone has shown promise, but data in pregnancy remains limited. ${ }^{27,40}$ In communities where these interventions are not available, the possibility of antepartum detoxification and other alternatives to methadone remain possibilities, and efforts should be made to expand access. ${ }^{41}$ Until studies confirm that these treatment modalities provide at least equivalent outcomes to methadone, and social and financial resources are provided to make them accessible to patients and physicians, methadone is likely to remain a highly used option for OUT. Consequently, we must commit ourselves to the pursuit of evidence-based rationales to help us understand and improve therapy for mothers with opioid use disorder.

\section{Conclusion}

Patients requiring methadone for OUT during pregnancy seem to achieve minimal, if any, decreases in their methadone dose 
after delivery. Furthermore, it was substantially less than the postpartum reduction of $50 \%$ recommended at the time of the study. Patients should be counseled that methadone for OUT during pregnancy may cause alterations in methadone requirements that make postpartum dose tapers challenging and should be counseled about alternatives to methadone.

\section{Note}

This paper was presented in poster format at the Annual Meeting of the Society of Maternal Fetal Medicine, Chicago, IL, on February 4, 2012.

\section{Conflict of Interest}

None of the authors have a conflict of interest.

\section{References}

1 Doberczak TM, Kandall SR, Friedmann P. Relationship between maternal methadone dosage, maternal-neonatal methadone levels, and neonatal withdrawal. Obstet Gynecol 1993;81(06):936-940

2 Malpas TJ, Darlow BA, Lennox R, Horwood LJ. Maternal methadone dosage and neonatal withdrawal. Aust N Z J Obstet Gynaecol 1995;35(02):175-177

3 Dashe JS, Sheffield JS, Olscher DA, Todd SJ, Jackson GL, Wendel GD. Relationship between maternal methadone dosage and neonatal withdrawal. Obstet Gynecol 2002;100(06):1244-1249

4 Berghella V, Lim PJ, Hill MK, Cherpes J, Chennat J, Kaltenbach K. Maternal methadone dose and neonatal withdrawal. Am J Obstet Gynecol 2003;189(02):312-317

5 Bakstad B, Sarfi M, Welle-Strand GK, Ravndal E. Opioid maintenance treatment during pregnancy: occurrence and severity of neonatal abstinence syndrome. A national prospective study. Eur Addict Res 2009;15(03):128-134

6 Lim S, Prasad MR, Samuels P, Gardner DK, Cordero L. High-dose methadone in pregnant women and its effect on duration of neonatal abstinence syndrome. Am J Obstet Gynecol 2009;200 (01):70.e1-70.e5

7 Drozdick J III, Berghella V, Hill M, Kaltenbach K. Methadone trough levels in pregnancy. Am J Obstet Gynecol 2002;187(05):1184-1188

8 Wolff K, Boys A, Rostami-Hodjegan A, Hay A, Raistrick D. Changes to methadone clearance during pregnancy. Eur J Clin Pharmacol 2005;61(10):763-768

9 Kaltenbach K, Berghella V, Finnegan L. Opioid dependence during pregnancy. Effects and management. Obstet Gynecol Clin North Am 1998;25(01):139-151

10 Department of Health and Human Services, Substance Abuse and Mental Health Services Administration. Results from the 2005 National Survey on Drug Use and Health: National Findings. Available at: https://www.dpft.org/resources/NSDUHresults2005. pdf. Accessed April 22, 2020

11 Jones HE, Johnson RE, O'Grady KE, Jasinski DR, Tuten M, Milio L. Dosing adjustments in postpartum patients maintained on buprenorphine or methadone. J Addict Med 2008;2(02):103-107

12 Albright B, de la Torre L, Skipper B, Price S, Abbott P, Rayburn W. Changes in methadone maintenance therapy during and after pregnancy. J Subst Abuse Treat 2011;41(04):347-353

13 Bogen DL, Perel JM, Helsel JC, et al. Pharmacologic evidence to support clinical decision making for peripartum methadone treatment. Psychopharmacology (Berl) 2013;225(02):441-451

14 Pace CA, Kaminetzky LB, Winter M, et al. Postpartum changes in methadone maintenance dose. J Subst Abuse Treat 2014;47(03): 229-232

15 Ostrea EM Jr., Brady M, Gause S, Raymundo AL, Stevens M. Drug screening of newborns by meconium analysis: a large-scale, prospective, epidemiologic study. Pediatrics 1992;89(01):107-113
16 Martin CE, Longinaker N, Terplan M. Recent trends in treatment admissions for prescription opioid abuse during pregnancy. J Subst Abuse Treat 2015;48(01):37-42

17 Pan IJ, Yi HY. Prevalence of hospitalized live births affected by alcohol and drugs and parturient women diagnosed with substance abuse at liveborn delivery: United States, 1999-2008. Matern Child Health J 2013;17(04):667-676

18 McCarthy JJ, Leamon MH, Parr MS, Anania B. High-dose methadone maintenance in pregnancy: maternal and neonatal outcomes. Am J Obstet Gynecol 2005;193(3, Pt 1):606-610

19 Burns L, Mattick RP, Lim K, Wallace C. Methadone in pregnancy: treatment retention and neonatal outcomes. Addiction 2007;102 (02):264-270

20 Jones HE, O'Grady KE, Malfi D, Tuten M. Methadone maintenance vs. methadone taper during pregnancy: maternal and neonatal outcomes. Am J Addict 2008;17(05):372-386

21 Luty J, Nikolaou V, Bearn J. Is opiate detoxification unsafe in pregnancy? J Subst Abuse Treat 2003;24(04):363-367

22 Dashe JS, Jackson GL, Olscher DA, Zane EH, Wendel GD Jr. Opioid detoxification in pregnancy. Obstet Gynecol 1998;92(05):854-858

23 Fischer G, Jagsch R, Eder H, et al. Comparison of methadone and slow-release morphine maintenance in pregnant addicts. Addiction 1999;94(02):231-239

24 Hartwig C, Haasen C, Reimer J, et al. Pregnancy and birth under maintenance treatment with diamorphine (heroin): a case report. Eur Addict Res 2008;14(02):113-114

25 Jones HE, Johnson RE, Jasinski DR, Milio L. Randomized controlled study transitioning opioid-dependent pregnant women from short-acting morphine to buprenorphine or methadone. Drug Alcohol Depend 2005;78(01):33-38

26 Jones HE, Johnson RE, Jasinski DR, et al. Buprenorphine versus methadone in the treatment of pregnant opioid-dependent patients: effects on the neonatal abstinence syndrome. Drug Alcohol Depend 2005;79(01):1-10

27 Hulse G, O'Neil G. Using naltrexone implants in the management of the pregnant heroin user. Aust N Z J Obstet Gynaecol 2002;42 (05):569-573

28 Christensen C. Management of chemical dependence in pregnancy. Clin Obstet Gynecol 2008;51(02):445-455

29 Kuschel CA, Austerberry L, Cornwell M, Couch R, Rowley RS. Can methadone concentrations predict the severity of withdrawal in infants at risk of neonatal abstinence syndrome? Arch Dis Child Fetal Neonatal Ed 2004;89(05):F390-F393

30 Nanovskaya TN, Deshmukh SV, Nekhayeva IA, Zharikova OL, Hankins GD, Ahmed MS. Methadone metabolism by human placenta. Biochem Pharmacol 2004;68(03):583-591

31 Hieronymus TL, Nanovskaya TN, Deshmukh SV, Vargas R, Hankins GD, Ahmed MS. Methadone metabolism by early gestational age placentas. Am J Perinatol 2006;23(05):287-294

32 Ahmed MS, Cemerikic B, Mou S, Agbas A. Effects of methadone use during pregnancy on human placental opioid receptors. Membr Biochem 1993;10(02):91-98

33 Cemerikic B, Zamah R, Ahmed MS. Opioid tolerance in human placenta due to in vitro methadone administration. J Pharmacol Exp Ther 1995;273(03):987-994

34 Center for Substance Abuse Treatment. Treatment Improvement Protocol (TIP) Series 43. DHHS Publication No. (SMA) 12-4214. Available at: https://www.ncbi.nlm.nih.gov/books/NBK82999/. Accessed April 22, 2020

35 Substance Abuse and Mental Health Services Administration. Clinical Guidance for Treating Pregnant and Parenting Women eith Opioid Use Disorder and Their Infants. Available at: https:// www.drugsandalcohol.ie/28957/1/Samsha_Clinical_guidance_for_treating-pregnant_and_parenting_women-with-opioid.pdf. Accessed April 22, 2020

36 Fitzsimons HE, Tuten M, Vaidya V, Jones HE. Mood disorders affect drug treatment success of drug-dependent pregnant women. J Subst Abuse Treat 2007;32(01):19-25 
37 Lejeune C, Simmat-Durand L, Gourarier L, Aubisson S; Groupe d'Etudes Grossesse et Addictions (GEGA). Prospective multicenter observational study of 260 infants born to 259 opiate-dependent mothers on methadone or high-dose buprenophine substitution. Drug Alcohol Depend 2006;82(03):250-257

38 Kakko J, Heilig M, Sarman I. Buprenorphine and methadone treatment of opiate dependence during pregnancy: comparison of fetal growth and neonatal outcomes in two consecutive case series. Drug Alcohol Depend 2008;96(1-2):69-78
39 Jones HE, Kaltenbach K, Heil SH, et al. Neonatal abstinence syndrome after methadone or buprenorphine exposure. $\mathrm{N}$ Engl J Med 2010;363(24):2320-2331

40 Hulse GK, O'Neil G, Arnold-Reed DE. Methadone maintenance vs. implantable naltrexone treatment in the pregnant heroin user. Int J Gynaecol Obstet 2004;85(02):170-171

41 Deck D, Carlson MJ. Access to publicly funded methadone maintenance treatment in two western states. J Behav Health Serv Res 2004;31(02):164-177 